\title{
ERROR ESTIMATES FOR THE APPROXIMATION OF FUNCTIONS BY CERTAIN INTERPOLATION POLYNOMIALS
}

\author{
STUART JOHN GOODENOUgh
}

In this thesis, we solve three distinct, yet related, problems in approximation theory. The problems are related in that they involve estimation of the exror incurred in replacing continuous functions by interpolation polynomials. Yet they are distinct in two fundamental ways. Firstly, we employ a different sequence of interpolation polynomials in each problem. Secondly, and more importantly, we obtain a different kind of error estimate in each problem. Indeed, one of the error estimates that we obtain is a complete asymptotic expansion, another is a pointwise error estimate, and the third is an asymptotic error estimate.

The importance of interpolation in modern approximation theory was guaranteed when the famous Hungarian mathematician, Leopold Fejer [2] employed a particular sequence of interpolation polynomials, called HermiteFejer interpolation polynomials, in presenting a new proof of the Weierstrass approximation theorem. His fundamental paper has stimulated research in two different directions. On the one hand, mathematicians have been interested in measuring the rate of convergence of Hermite-Fejer interpolation. On the other hand, mathematicians have searched for other interpolation polynomials which will converge uniformly to the given continuous function. Of particular interest are the so-called $(0,1,2,3)-$ interpolation polynomials and quasi-Hermite-Fejér interpolation

Received 29 August 1985. Thesis submitted to University of Newcastle, February 1985. Degree approved August 1985. Supervisors:

Professor J. R. Giles, Dr. T. M. Mills.

Copyright Clearance Centre, Inc. Serial-fee code: 0004-9727/86 $\$ \mathrm{~A} 2.00+0.00$. 
polynomials. Chapter 1 serves to introduce these interpolation polynomials and to provide a unifying theme for our estimation problems.

Our first problem deals with the approximation of a Lipschitz class of functions by Hermite-Fejér interpolation polynomials. As an introduction to this problem, a theorem due to Bojanic $[1, p .70]$ can be modified to show that the maximum interpolation error incurred in approximating the Lipschitz class by Hermite-Fejér interpolation polynomials is asymptotically like $\frac{\log n}{n}$. Our task is more ambitious in that we seek to obtain the complete asymptotic expansion for the maximum interpolation error. Indeed, as far as asymptotic estimates are concerned, a complete asymptotic expansion is the best possible result. As a byproduct of our expansion, we find that the first term is $\frac{2}{\pi} \frac{\log n}{n}$, which adds precision to Bojanic's result. We devote Chapters 2 and 3 to these matters; these are published in Goodenough [4]. A comparison of the complete asymptotic expansions for the maximum interpolation error and for the Lebesgue constant of oxder $n$ then enables us to establish a close relationship between these two quantities; a relationship which is totally unexpected. This discovery is published in Goodenough [3].

Our second problem deals with the approximation of a continuous function by $(0,1,2,3)$-interpolation polymomials. Although various error estimates have been obtained by different authors, including uniform estimates, pointwise estimates, and an asymptotic estimate by Goodenough and Mills [5], none of these estimates reflects the interpolation conditions. In other words, none of these estimates vanishes at the nodes of interpolation. Our task is to obtain a new pointwise error estimate which reflects the interpolation conditions. We solve this problem in Chapter 4; this work has been published in Goodenough and Mills [7].

Our third problem is concerned with the approximation of a class of Lipschitz functions by quasi-Hermite-Fejer interpolation polynomials. We obtain an asymptotic estimate (for the error incurred) which reflects the interpolation conditions. This estimate in turn enables us to solve a best constant problem. This is the content of Chapter 5 and has been published in Goodenough and Mills [6]. 


\section{References}

[1] R. Bojanic, "A note on the precision of interpolation by HermiteFejer polynomials", Proceedings of the Conference on constructive theory of functions, Budapest 1969, 69-76 (Akadémiai Kiadó, Budapest, 1972).

[2] I. Fejer, "Ueber Interpolation", Nachr.Akad.Wiss.Göttingen Math.-Phys. K1. (1916), 66-91.

[3] S.J. Goodenough, "A link between Lebesgue constants and HermiteFejér interpolation", Bull.Austral.Math.Soc. 33 (1986), 207-218.

[4] S.J. Goodenough, "The complete asymptotic expansion for the degree of approximation of Lipschitz functions by Hermite-Fejér interpolation polynomials", J.Approx. Theory 44 (1985), 325-342.

[5] S.J. Goodenough and T.M. Mills, "The asymptotic behaviour of certain interpolation polynomials", J.Approx. Theory 28 (1980), 309-316.

[6] S.J. Goodenough and T.M. Mills, "Asymptotic estimates for quasi-Hermite-Fejêr interpolation", Acta Math.Acad.Sci.Hungar. 38 (1981), 151-155.

[7] S.J. Goodenough and T.M. Mills, "On interpolation polynomials of the Hermite-Fejer type II", Bull.Austral.Math.Soc. 23 (1981), 283-291.

\footnotetext{
Department of Mathematics, Statistics

and Computer Science,

University of Newcastle, Newcastle, N.S.W. 2308,

Australia.
} 